\title{
Control Law Design for Perching an Agile MAV with Articulated Wings
}

\author{
Animesh Chakravarthy ${ }^{*}$ Aditya Paranjape ${ }^{\dagger}$ and Soon-Jo Chung ${ }^{\ddagger}$
}

\begin{abstract}
This paper explores the use of variable wing dihedral and variable wing twist (in conjunction with a conventional horizontal elevator) to control an aircraft performing a perching maneuver. A choice of controller architecture wherein the dihedral is employed in the forward path and the elevator and twist are employed in the feedback path, is considered. The aircraft is modeled as a multivariable linear time-varying system. A specific perching trajectory is considered; and the open-loop aircraft is longitudinally unstable for a segment of this perching trajectory and lateral-directionally unstable for the entire perching trajectory. A multivariable time-varying controller is designed to efficiently stabilize the aircraft as well as reject longitudinal-lateral-directional wind disturbances, while closely tracking the reference perching trajectory.
\end{abstract}

\section{Introduction}
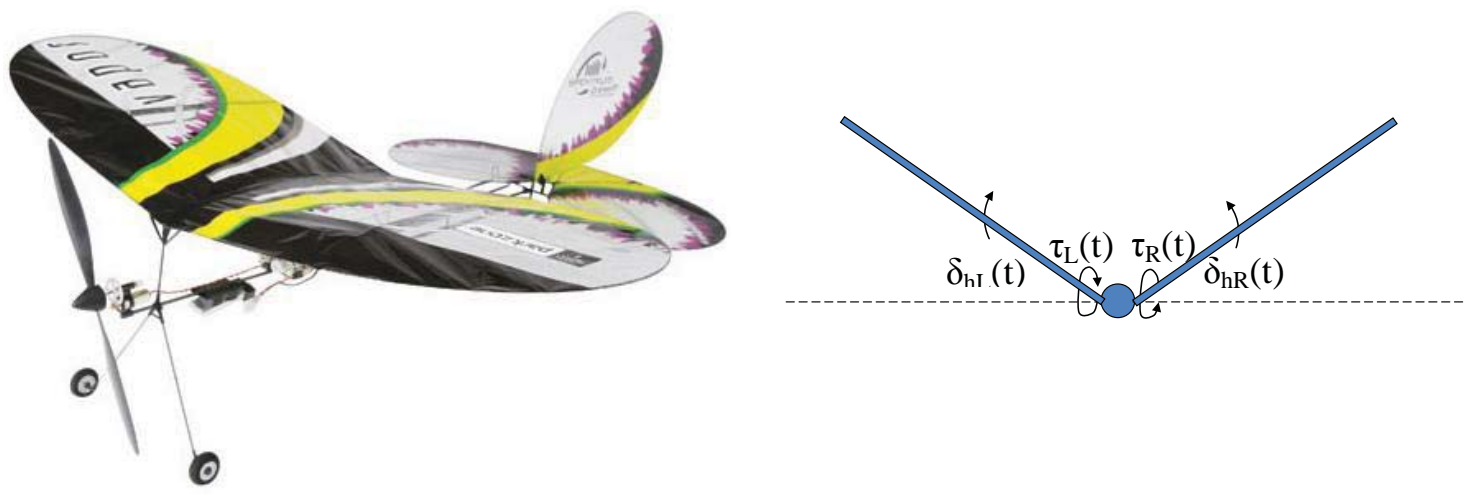

Figure 1. (a) ParkZone Vapor: The aircraft model considered in this paper (modeled without the vertical tail) (b) Schematic representation of variable dihedral and twist

Currently, there is considerable interest in the aerospace community to learn and mimic the flight of birds and insects. Micro air vehicles (MAVs) of the future will have the ability to perform high agility maneuvers within constrained and cluttered environments. The performance of such complex maneuvers requires a combination of open and closed loop capabilities - however the performance achievable in the closed loop is obviously dependent upon the capabilities of the airframe, the actuator dynamics, etc. In this paper, the focus is on the design of a guidance and control law for perching of a MAV. This paper forms part of a series of efforts ${ }^{1,2}$ in studying the flight mechanics and control of tail-less, articulated wing aircraft. Paranjape and Chung ${ }^{1}$ demonstrate the utility of asymmetric dihedral for yaw control and the use of symmetric dihedral for longitudinal maneuvers, while Paranjape $e t a l^{2}$ address the effects of wing flexibility.

\footnotetext{
${ }^{*}$ Research Assistant Scientist, Dept. of Mechanical and Aerospace Engineering, University of Florida Research and Engineering Education Facility (UF-REEF), Shalimar, FL; Senior Member, AIAA; animesh@reef.ufl.edu.

${ }^{\dagger}$ Doctoral Candidate, Dept. of Aerospace Engineering, University of Illinois at Urbana Champaign (UIUC), Urbana, IL; Student Member, AIAA; paranja2@illinois.edu

${ }^{\ddagger}$ Assistant Professor, Dept. of Aerospace Engineering, University of Illinois at Urbana Champaign (UIUC), Urbana, IL; Senior Member, AIAA; sjchung@illinois.edu.
} 
In particular, this series of papers consider a new wing concept motivated by avian wings. The focus of these papers is on a wing which can be rotated at the root to generate variable dihedral and twist. Both - the dihedral angles as well as the twist angles generated on the port and starboard wings need not be equal. The aircraft considered here is a modified version of the one depicted in Figure 1(a). Figure 1(b) is a schematic representation to demonstrate the presence of variable dihedral and twist on this aircraft. Aerodynamic contributions of the fuselage and the thrust have been ignored with the understanding that their effects can be readily incorporated into the control law design. Also, while it is true that these wings do not flap during the perching maneuver (similar to birds), they could in general, be flapping during other flight phases.

Fixed and flapping wing MAVs have been extensively studied in the literature. Mueller ${ }^{4}$ provides an excellent compendium of papers for some of the work done in this area until the beginning of the 21st century. Seigler et al, ${ }^{5}$ Sanders et al, ${ }^{6}$ Bowman et al, ${ }^{7}$ Tidwell et al, ${ }^{8}$ Bye and McClure, ${ }^{9}$ Abdulrahim and Lind ${ }^{10}$ are among several of the papers in the last decade that discuss various aspects of morphing aircraft. Costello and Webb ${ }^{11}$ demonstrated that articulated wing MAVs, hinged at the root, could reduce gust sensitivity. Wickenheiser and Garcia ${ }^{12,13}$ studied the dynamics of morphing aircraft and demonstrated perching using, among other forms of articulation, variable wing incidence. Reich et al ${ }^{14}$ experimentally studied the aerodynamic performance of a wing of variable incidence for perching. Roberts et $a l^{21}$ examined the perching problem of a fixed-wing aircraft from controllability aspects. Desbiens and Cutkosky ${ }^{22}$ examined the problem of perching on vertical surfaces. The lateral stability and control of birds, and in particular, the role of wing dihedral, have been studied extensively by Sachs. ${ }^{15-17}$ These papers have demonstrated that for air vehicles whose size and speed are similar to those of birds, wings are sufficient to provide lateral stability, thereby reducing, if not eliminating altogether, the need for a vertical tail. This paper is meant to demonstrate the use of dihedral as well as wing twist for perching maneuvers in the presence of both longitudinal as well as lateral-directional wind disturbances.

\section{Influence of variable dihedral and twist}

The effect of having variable dihedral and twist on the left and right wings is to modify the effective angle of attack experienced at any given section on the wing span. In this paper, we assume that $C_{l}(y)$ and $C_{d}(y)$ (where $y$ represents a section along the wing span) to be given by the following expressions: ${ }^{18}$

$$
\begin{gathered}
C_{l}(y)=0.225+1.58 \sin (2.13 \alpha(y)-7.2) \\
C_{d}(y)=1.92-1.55 \cos (2.04 \alpha(y)-9.82)+1 /(0.95 \pi A R) C_{l}^{2}
\end{gathered}
$$

where $\alpha(y)$ indicates the angle of attack experienced at span station $y$ and $A R$ indicates the aspect ratio. The wing planform is taken to be rectangular with half span of $0.375 \mathrm{~m}$ and a chord length of $0.146 \mathrm{~m}$.

For given free stream velocity components $\left[\begin{array}{lll}u & v & w\end{array}\right]^{T}$ in the body frame, the effect of variable dihedral $\delta_{h}$ is to transform them into local velocity components $\left[\begin{array}{lll}u_{L O C} & v_{L O C} & w_{L O C}\end{array}\right]^{T}$ as per the following transformation:

$$
\left[\begin{array}{c}
u_{L O C} \\
v_{L O C} \\
w_{L O C}
\end{array}\right]=\left[\begin{array}{ccc}
1 & 0 & 0 \\
0 & \cos \left(\delta_{h}\right) & -\sin \left(\delta_{h}\right) \\
0 & \sin \left(\delta_{h}\right) & \cos \left(\delta_{h}\right)
\end{array}\right]\left[\begin{array}{c}
u \\
v \\
w
\end{array}\right]
$$

In a similar fashion, the effect of variable twist $\tau$ is to transform the free stream velocity components into local velocity components as per the following transformation given below:

$$
\left[\begin{array}{c}
u_{L O C} \\
v_{L O C} \\
w_{L O C}
\end{array}\right]=\left[\begin{array}{ccc}
\cos (\tau) & 0 & -\sin (\tau) \\
0 & 1 & 0 \\
\sin (\tau) & 0 & \cos (\tau)
\end{array}\right]\left[\begin{array}{c}
u \\
v \\
w
\end{array}\right]
$$

From the above expressions, one can determine that with $\beta_{F S}=0$ and twist angle $\tau=0$, for a given free stream angle of attack $\alpha_{F S}$, the effect of dihedral $\delta_{h}$ is to transform the same into an $\alpha(y)$ given by:

$$
\alpha(y)=\arctan \left(\tan \left(\alpha_{F S}\right) \cos \left(\delta_{h}\right)\right)
$$


Similarly, with $\beta_{F S}=0$ and dihedral angle $\delta_{h}=0$, the effect of twist $\tau$ is to transform $\alpha_{F S}$ into an $\alpha(y)$ given by:

$$
\alpha(y)=\arctan \left[\frac{\sin (\tau)+\tan \left(\alpha_{F S}\right) \cos (\tau)}{\cos (\tau)-\tan \left(\alpha_{F S}\right) \sin (\tau)}\right]
$$

Finally, with $\beta_{F S}=0$, the effect of simultaneous non-zero values of both $\delta_{h}$ as well as $\tau$ is to transform $\alpha_{F S}$ into an $\alpha(y)$ given by:

$$
\alpha(y)=\arctan \left[\frac{\sin (\tau)+\tan \left(\alpha_{F S}\right) \cos \left(\delta_{h}\right) \cos (\tau)}{\cos (\tau)-\tan \left(\alpha_{F S}\right) \cos \left(\delta_{h}\right) \sin (\tau)}\right]
$$

In the above expressions, the effects of aircraft angular rates $p, q, r$ as well as rates of change of dihedral and/or twist have been neglected. Using these expressions, one can compute the total aerodynamic force and moment co-efficients. The aerodynamic contributions of the fuselage have been ignored in this study only the effects of the wing and the horizontal tail have been taken into account.

The local aerodynamic force at a station is given by the vector sum of the lift and the drag, with components written in the body frame:

$$
\mathbf{d} \mathbf{F}=0.5 \rho\|\mathbf{V}(y)\|^{2} c\left(C_{l} \mathbf{l} /\|\mathbf{l}\|+C_{d} \mathbf{d} /\|\mathbf{d}\|\right) d y
$$

where

$$
\mathbf{l}=R\left[\begin{array}{lll}
0 & 1 & 0
\end{array}\right]^{T} \quad X \quad \mathbf{V}(y) /\|\mathbf{V}(y)\|
$$

where $R$ corresponds to the rotation matrix given in Eqn (3), $X$ denotes the vector cross-product and

$$
\mathbf{d}=-\mathbf{V}(y) /\|\mathbf{V}(y)\|
$$

Similarly, the local aerodynamic moment at a station is given by

$$
\mathbf{d} \mathbf{M}=\mathbf{r} \quad X \quad \mathbf{D F}+0.5 \rho\|\mathbf{V}(y)\|^{2} c C_{m_{a c}} R\left[\begin{array}{lll}
0 & 1 & 0
\end{array}\right]^{T} d y
$$

where $r$ is the position vector of the aerodynamic center of the station.
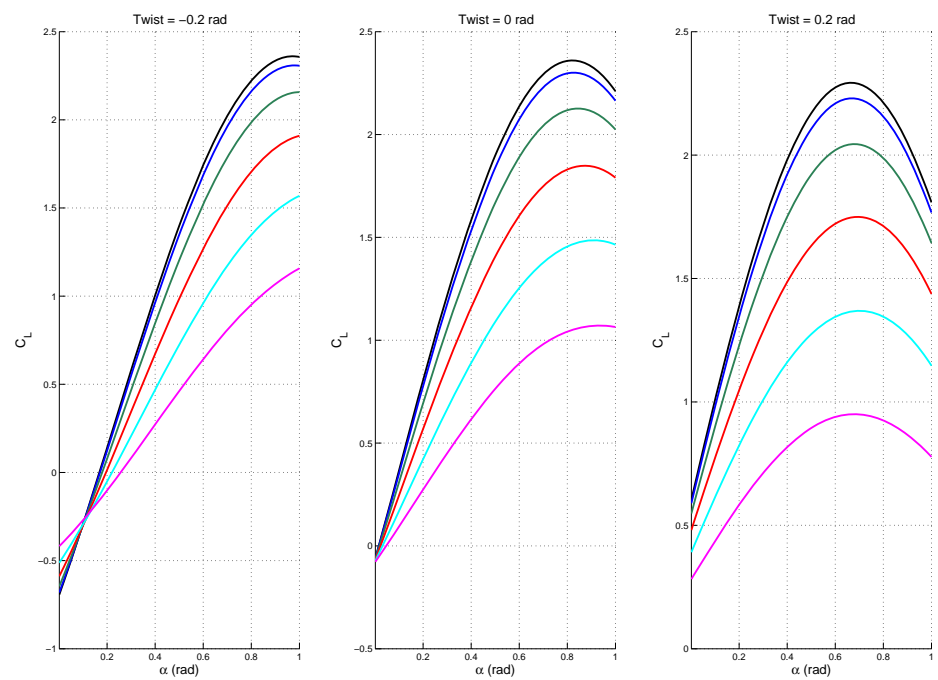

Figure 2. $C_{L}$ vs. $\alpha$ for different values of symmetric dihedral and symmetric twist (a) Twist $=-0.2 \mathrm{rad}(\mathrm{b})$ Twist $=0$ $\mathrm{rad}(\mathrm{c})$ Twist $=0.2 \mathrm{rad}$. In (a-c), magnitudes of dihedral are 0 (black), $0.2 \mathrm{rad}$ (blue), $0.4 \mathrm{rad}(\mathrm{green}), 0.6 \mathrm{rad}(\mathrm{red}), 0.8$ $\operatorname{rad}$ (cyan), 1 rad (magneta)

Figure 2 shows three graphs of $C_{L}$ versus $\alpha$ for different values of twist corresponding to $\tau=-0.2 \mathrm{rad}$, $\tau=0 \mathrm{rad}$ and $\tau=0.2 \mathrm{rad}$. In each of these three graphs, the effects of the different magnitudes of dihedral angles are brought out. Note that the $\alpha$ represented on the abscissa of these plots is actually the free stream angle of attack. These curves demonstrate that moving from negative values of twist to positive values of 
twist has the effect of increasing the value of $C_{L}$ at zero free stream angle of attack. For zero twist, there is negligible influence of dihedral angle on the $C_{L}$ at $\alpha=0$, while with non-zero twist, $C_{L}$ at $\alpha=0$ does change with different values of dihedral. For $\tau=-0.2 \mathrm{rad}$, increasing magnitudes of dihedral have the effect of increasing the $C_{L}$ at $\alpha=0$, while for $\tau=0.2 \mathrm{rad}$, increasing magnitudes of dihedral have the effect of decreasing $C_{L}$ at $\alpha=0$. Finally, for both $\tau=0$ as well as $\tau=0.2 \mathrm{rad}$, the $C_{L}$ curve gets lowered with increasing magnitudes of dihedral and this trend also holds true for the $\tau=-0.2$ rad case, for values of $\alpha$ greater than $0.1 \mathrm{rad}$.

Figure 3 then shows three graphs of $C_{D}$ versus $\alpha$, again for values of twist corresponding to $\tau=-0.2 \operatorname{rad}$, $\tau=0 \mathrm{rad}$ and $\tau=0.2 \mathrm{rad}$. Again, the effects of different magnitudes of dihedral angles are demonstrated. In this case, varying dihedral values do not influence the drag experienced at $\alpha=0$ for any of the three twist values. In general, increasing values of dihedral have the effect of lowering the drag co-efficient for the non-negative values of $\tau$ demonstrated in the figures, while for the negative $\tau$ shown, the same is true for values of $\alpha$ greater than about $0.1 \mathrm{rad}$.
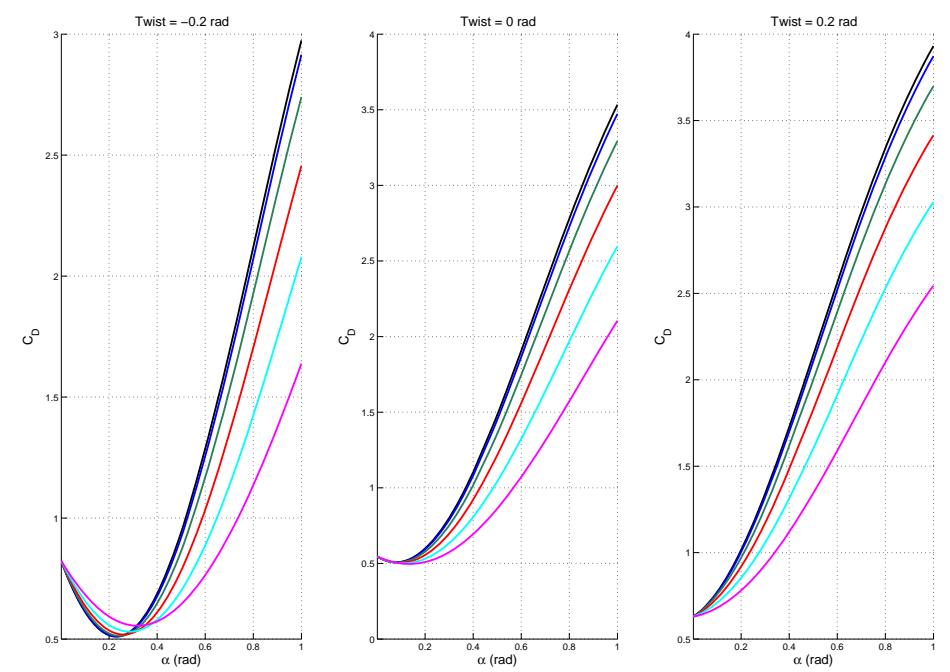

Figure 3. $C_{D}$ vs. $\alpha$ for different values of symmetric dihedral and symmetric twist (a) Twist $=-0.2 \mathrm{rad}(\mathrm{b})$ Twist $=0$ $\mathrm{rad}(\mathrm{c})$ Twist $=0.2 \mathrm{rad}$. In (a-c), magnitudes of dihedral are 0 (black), $0.2 \mathrm{rad}$ (blue), $0.4 \mathrm{rad}(\mathrm{green}), 0.6 \mathrm{rad}(\mathrm{red}), 0.8$ rad (cyan), 1 rad (magneta)

One of the benefits of having variable dihedral capability is that it provides us the ability to trim the aircraft at independent values of velocity $V$ and angle of attack $\alpha$. Since the (simplified form of ) a vertical trim equation is simply given by $0.5 \rho V^{2} S C_{L}=W$ (where $W$ stands for the weight of the aircraft which is fixed and given), for conventional aircraft, the desire to trim at a particular value of $V$ automatically fixes $C_{L}$, which in turn fixes the value of $\alpha$. However, for a variable dihedral aircraft, since one can get the same $C_{L}$ for different values of $\alpha$ simply by varying the dihedral, this extra degree of freedom is available. Figure 4 demonstrates the different trim values of $V$ and $\alpha$ obtainable as a function of the dihedral angle $\delta_{h}$ and keeping the elevator angle $\delta_{e}$ constant and equal to a value of $-0.2 \mathrm{rad}$. We see that in general, lower magnitudes of dihedral enable trimming the aircraft at lower velocities, while higher magnitudes of dihedral necessiate trimming the aircraft at higher velocities. An opposite trend is seen as far as trim angles of attack are concerned, i.e. lower magnitudes of dihedral require higher trim angles of attack while higher magnitudes of dihedral require lower trim angles of attack, which is along expected lines. Note that the trim values demonstrated in this figure are for symmetrical values of dihedral, i.e. $\delta_{h_{L}}=\delta_{h_{R}}$. The horizontal tail and symmetric dihedral combination can thus serve with great utility for the purpose of efficient control of perching maneuvers, in the absence of cross winds during the perching trajectory. In the presence of cross winds during the perching trajectory, the asymmetric twist can be used for control to reject the ensuing non-zero values of sideslip angle. 

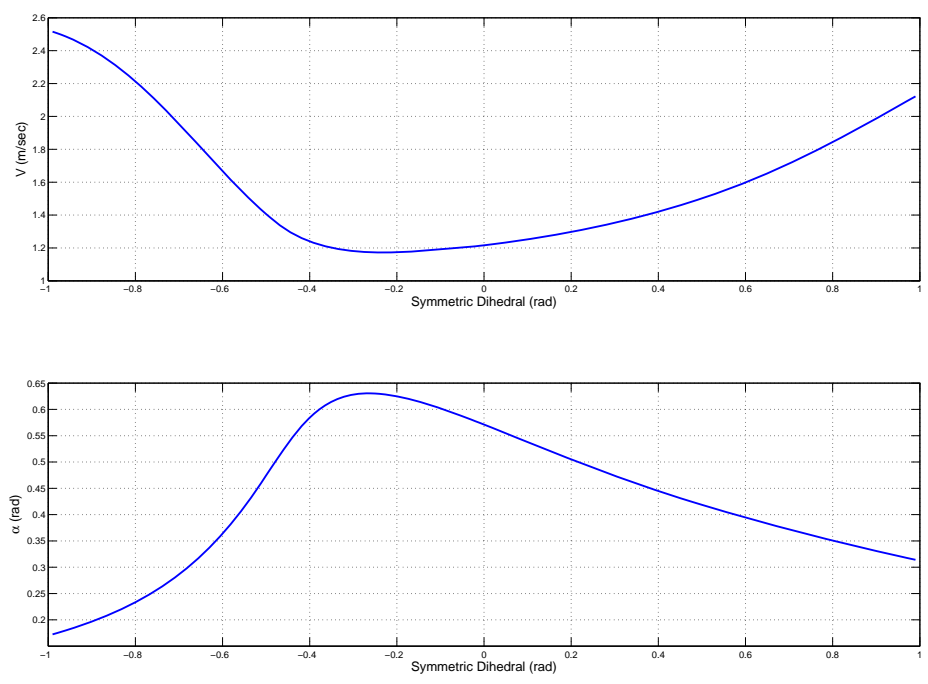

Figure 4. Trim values of velocity and angle of attack as a function of dihedral angle

\section{Perching Trajectory and Aircraft Dynamics}

\section{III.A. Perching Trajectory}

Perching is, by and large, a bioinspired maneuver. On lines similar to those of soaring birds, a perching aircraft (with a comparatively low thrust-to-weight ratio) must fly below the point of landing and then perform a pull-up just before actually landing - the purpose of the undershoot being to use gravity to drain away the last of its kinetic energy. ${ }^{19}$ While it is obviously true that such an undershoot is impossible to perform on flat ground, it can be very effectively exploited for landing on an elevated platform, over a short landing trajectory. Such elevated platforms are quite commonly available in both natural as well as urban environments (for instance in a building ledge, power line or a tree branch) and can be ideally used for the aircraft to perform extended surveillance missions - the reason these surveillance missions can be of extended duration is because once the aircraft perches, it can continue to survey the area without consuming fuel for flight. ${ }^{13}$

The choice of an optimal perching trajectory has been studied in the literature. ${ }^{12,13}$ For low thrust to weight ratios, such trajectories comprise of an undershoot and therefore the whole perching trajectory is essentially divided into two parts: a dive phase followed by a climb phase. During the dive phase, the flight path angle is naturally negative - it subsequently becomes positive during the climb phase. At the landing point, the pitch attitude angle necessarily needs to lie between $0 \mathrm{deg}$ and $90 \mathrm{deg}$ - this is required in order to enable the aircraft to perch on its landing struts. Furthermore, the final velocity is required to be only a small fraction of its initial velocity. While Wickenheiser and Garcia ${ }^{12}$ construct an optimal morphing trajectory that attempts to minimize both the undershoot as well as the distance from the landing site required to start the maneuver, the focus of this paper is not on determining such an optimal trajectory; but rather to pick out a reference perching trajectory that has qualitative features that are akin to a good perching trajectory (including the presence of an undershoot, short perching time, low final velocity, etc.) and then design a closed loop controller so as to enable the aircraft to follow this reference perching trajectory as closely as possible even in the presence of disturbances (both longitudinal as well as lateral).

With this view in mind, the reference perching trajectory is first constructed on a vertical plane (the $X Z$ plane containing a sequence of points $\left.\left(x_{1}, z_{1}\right),\left(x_{2}, z_{2}\right), \ldots,\left(x_{n}, z_{n}\right)\right)$. By keeping a fixed duration $T$ for the perching trajectory, these points are further written out as $\left(x_{1}\left(t_{1}\right), z_{1}\left(t_{1}\right)\right),\left(x_{2}\left(t_{2}\right), z_{2}\left(t_{2}\right)\right), \ldots,\left(x_{n}\left(t_{n}\right), z_{n}\left(t_{n}\right)\right)$. Then using the expressions $\dot{x}(t)=V(t) \cos (\gamma(t))$ and $\dot{z}(t)=V(t) \sin (\gamma(t))$, the reference perching trajectory is finally written out as a sequence of points on the $(V, \gamma)$ plane, i.e. as a set of time-indexed points $\left(V_{1}\left(t_{1}\right), \gamma_{1}\left(t_{1}\right)\right),\left(V_{2}\left(t_{2}\right), \gamma_{2}\left(t_{2}\right)\right), \ldots,\left(V_{n}\left(t_{n}\right), \gamma_{n}\left(t_{n}\right)\right)$. The controller is designed to follow a reference trajectory 


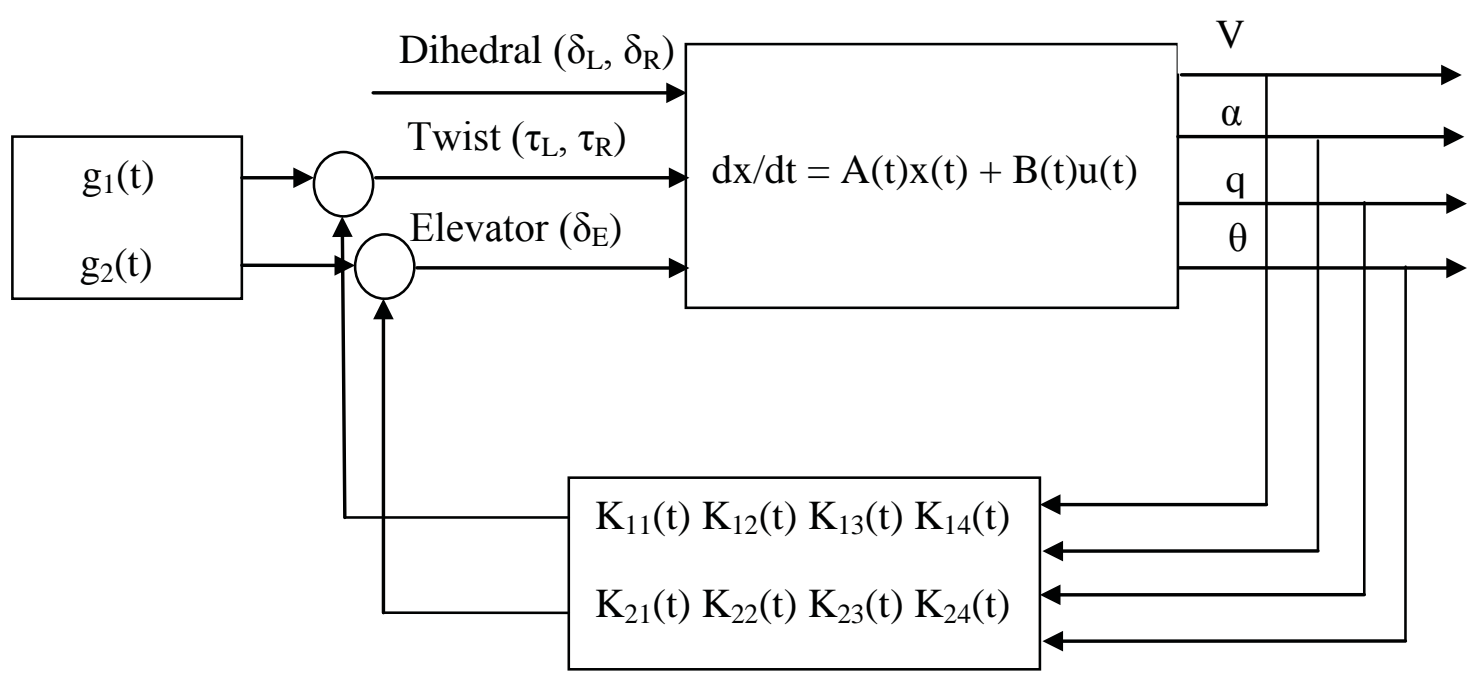

Figure 5. Control Law schematic

presented in this form.

A series of trim points for the aircraft that lie reasonably close to the reference perching trajectory (on the $(V, \gamma)$ plane) are then extracted. It turns out that this sequence of trim points lies naturally close to a varying (symmetric) dihedral trajectory that varies from approximately -1 deg to approximately -0.4 deg. Referring to Figure 4, one can see that this qualitatively makes sense since such a dihedral trajectory indeed corresponds to decreasing values of trim velocity. About this sequence of trim values (corresponding to varying dihedral), linear models of the aircraft are extracted. These individual models are then put together into the form $\dot{x}=A(t) x(t)+B(t) u(t)$ to generate an LTV (Linear Time-Varying) model. In other words, the original nonlinear aircraft model is linearized about a time-varying trajectory to generate a set of linear structures with time-dependent coefficients (See Chakravarthy et $a l^{24}$ for an analysis of time-varying dynamics of a morphing MAV). This linearization is distinct from the state-dependent coefficient factorization wherein the nonlinear equations are represented as linear structures with state-dependent coefficients (See Chang and Chung ${ }^{25}$ for an example). This thus forms the motivation for having the controller structure shown in Figure 5 - a variable (symmetric) dihedral trajectory is applied that keeps the aircraft reasonably close to (but not exactly on) the reference perching trajectory; the elevator and the twist are controlled in the feedback paths to keep the aircraft more closer to the perching trajectory as well as reject wind disturbances that may hit the aircraft. Furthermore, the fact that the left and right twist angles are controlled independently of each other enables them to be effectively used in either a symmetric fashion (to reject longitudinal wind disturbances), or in an asymmetric fashion (to reject lateral wind disturbances).

\section{III.B. Aircraft Dynamics}

Before emabarking on the controller design for the LTV model, we take a look at the open-loop dynamics of the aircraft. Note that for time-varying systems, the eigenvalues do not give any indication of stability whatsoever - indeed, presence of the real part of the eigenvalues (at each time instant) in the left half of the s-plane is neither a necessary nor a sufficient condition for stability of the LTV system. Furthermore, for such systems, the presence (or lack of) controllability cannot be inferred from the controllability properties of the LTI system taken pointwise in time. For stability issues, we therefore look at the state transition matrix of the LTV system. The state transition matrix of the unforced LTV system $\dot{x}=A(t) x(t)$ is represented by the matrix $\Phi\left(t, t_{0}\right)$ which is defined to be such that the solution of the unforced system can be written as $x(t)=\Phi\left(t, t_{0}\right) x\left(t_{0}\right)$. The state transition matrix is computed by solving the system of equations given by $\dot{\Phi}\left(t, t_{0}\right)=A(t) \Phi\left(t, t_{0}\right), \Phi\left(t_{0}, t_{0}\right)=I$ where $I$ represents the identity matrix. By looking at the boundedness (or lack thereof) of the state transition matrix for different $\tau$, the stability (or lack thereof) of the system 

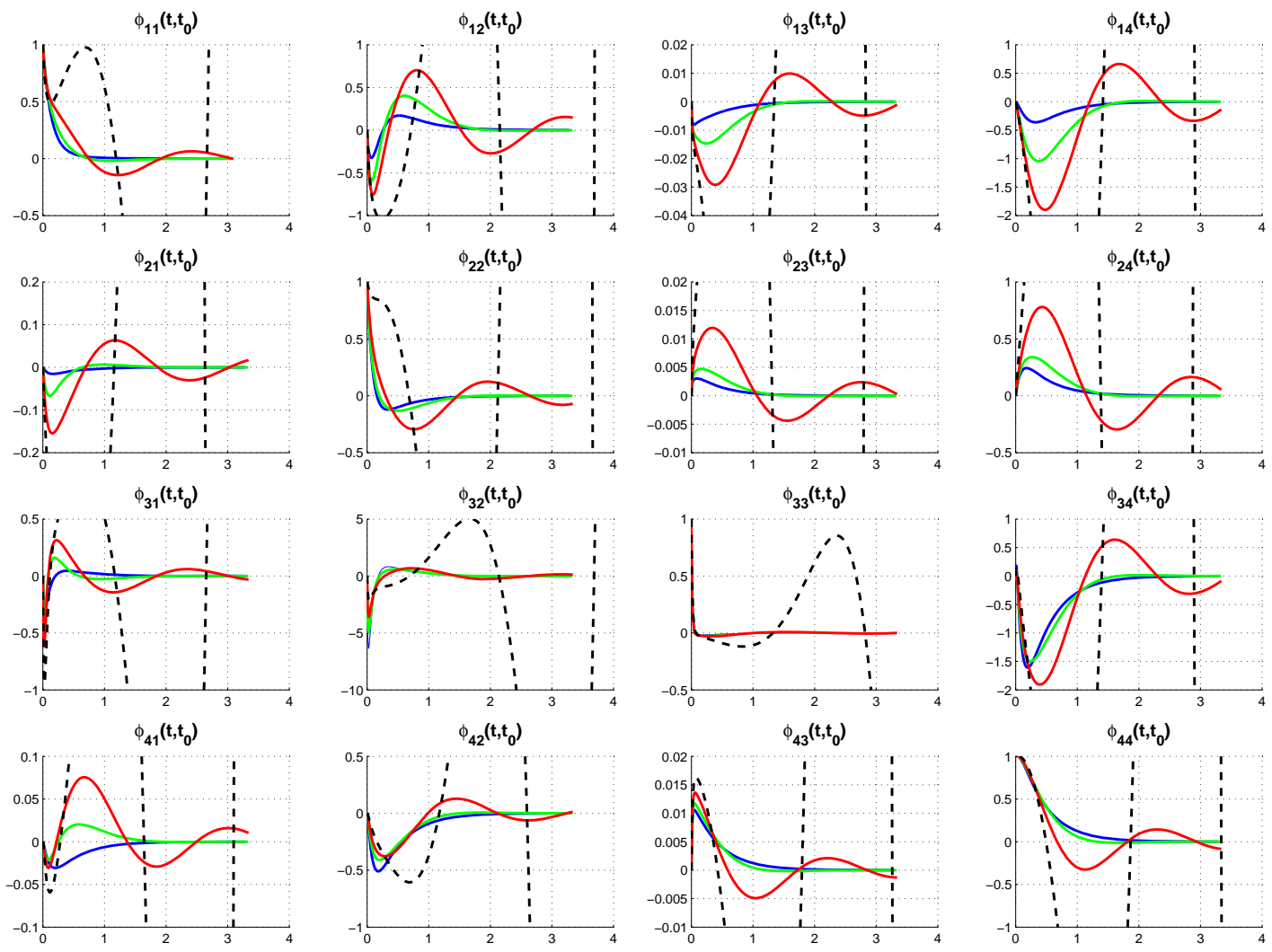

Figure 6. Elements of State Transition Matrix $\Phi\left(t, t_{0}\right)$ at different times during a symmetric dihedral trajectory, for $t_{0}=0$ (blue), $t_{0}=10 \mathrm{sec}$ (green), $t_{0}=17 \mathrm{sec}$ (red) and $t_{0}=25 \mathrm{sec}$ (dashed black). The abscissa in all these plots demonstrates $t-t_{0}$. For $t_{0}=25 \mathrm{sec}$, the unstable nature of the state transition matrix is demonstrated.

can be inferred.

Figure 6 demonstrates the elements of the state transition matrix (longitudinal axis only) of the aircraft being discussed in this paper, for several different values of $t_{0}$. Note that computing the state transition matrix for different values of $t_{0}$ corresponds to perturbing the system at different times along the perching trajectory. The figure shows that the longitudinal state transition matrix comprises of bounded elements for $\Phi(t, 0), \Phi(t, 10)$ and $\Phi(t, 17)$ but becomes unbounded in the case of $\Phi(t, 25)$. Note that since this system possesses time-varying dynamics, therefore the behavior of the system in response to perturbations hitting the system at different times, can be different. Figure 6 shows that this is indeed the case in the context of the aircraft being considered in this paper. A perturbation that hits the aircraft at $17 \mathrm{sec}$, for instance causes larger overshoots in the system response as compared to the case of a perturbation (of the exact same magnitude) hitting the aircraft at $10 \mathrm{sec}$. In turn, the response of the aircraft to a perturbation at $10 \mathrm{sec}$ is larger than the response to a perturbation at 0 sec. There is thus a progressive deterioration in the open-loop behavior of the longitudinal system as it progresses in time, along the perching trajectory. Furthermore, not only is the performance of the longitudinal open-loop system deteriorating along the perching trajectory; but the stability behavior is deteriorating too. The open-loop system is actually longitudinally stable for an initial part of the reference perching trajectory, and is subsequently longitudinally unstable for the latter part of the reference perching trajectory. We point out however that stability (wherever it exists) is not necessarily global, as far as the original nonlinear system is concerned. However, as far as lateral perturbations are concerned, the entire perching trajectory is unstable with respect to these perturbations.

Since the perching trajectory is unstable for large segments of time, a feedback controller of the structure shown in Figure 5 is attempted. It needs to be verified beforehand however, whether the LTV system 
is indeed controllable with the combination of elevator and twist. Furthermore, given that the perching trajectory selected has been found to be laterally unstable and that we are using variable dihedral only in a symmetric fashion, it is crucial to check for the controllability of the lateral states with respect to asymmetric twist inputs. When considering LTV systems, the notion of controllability (as known for LTI systems) splits up into the finer notions of complete controllability, total controllability and uniform controllability ${ }^{20}$ - these separate notions have been developed to cater to the fact that the relative influence of the control inputs on the state variables can change with time in LTV systems. Of these three different notions, uniform controllability is the strongest form of controllability; it requires that there exist a control at every single point in time (during the entire duration of the perching trajectory), that can bring a perturbed state from anywhere on the state space to the origin. One of the tests for uniform controllability of LTV systems requires checking the time-varying matrix $C(t)=\left[C_{1}(t)\left|C_{2}(t)\right| \ldots \ldots . \mid C_{n-1}(t)\right]$, where $C_{1}(t)=B(t)$ and $C_{i+1}(t)=A(t) C_{i}(t)-\dot{C}_{i}(t)$. The system is then uniformly controllable if and only if $C(t)$ is of full rank at each time $t$ along the perching trajectory. Using this condition, we verified that the aircraft being considered in this paper is indeed uniformly controllable through the entire duration of the perching trajectory.

\section{Control Laws for Perching}

\section{IV.A. Control Strategy}

In this section, we perform a time-varying optimal control law design to track the reference perching trajectory discussed in the previous section. Initially, only the longitudinal dynamics are considered and the objective of the controller is to both stabilize the aircraft during the longitudinally unstable segment of the perching trajectory, as well as to track the reference perching trajectory after rejecting longitudinal wind gusts, if any. Subsequently, the lateral dynamics too are appended onto the system and a lateral controller is designed whose objective is to stabilize the aircraft during the entire duration of the perching trajectory (since the entire perching trajectory is laterally unstable, as discussed in the previous section) as well as reject lateral wind gusts, if any. The presence of full-state feedback is assumed. The aircraft is required to perform the perch from an initial height of $100 \mathrm{~m}$ to a final height of $5 \mathrm{~m}$ over a duration of 40 seconds. The aircraft dihedral (symmetrically applied in the feedforward path) is varied in a continous fashion only over the first 30 seconds of the perching trajectory - this duration corresponding to the dive-phase of the trajectory before the start of the undershoot. For the final portion of the trajectory corresponding to the undershoot and climb, the dihedral is kept fixed at the final value it had just before the start of the undershoot. Discontinous changes in dihedral are avoided throughout the maneuver.

For the LTV system $\dot{x}=A(t) x(t)+B(t) u(t), y(t)=C x(t)$, where $x=\left[\begin{array}{llll}V & \alpha & q & \theta\end{array}\right]^{T}$ is the vector of longitudinal states, $u=\left[\begin{array}{lll}\delta_{e} & \tau_{L} & \tau_{R}\end{array}\right]$ is the vector of controls and $y=\left[\begin{array}{ll}V & \gamma\end{array}\right]^{T}$ is the vector of outputs, the controller is designed to minimize the following performance index: ${ }^{23}$

$$
J=\frac{1}{2} e(T)^{T} F e(T)+\frac{1}{2} \int_{t_{0}}^{T}\left[(z(t)-y(t))^{T} Q(t)(z(t)-y(t))+u(t)^{T} R(t) u(t)\right] d t
$$

where $z(t)$ indicates the reference perching trajectory on the $(V, \gamma)$ plane, $T$ indicates the final perching time, $R(t)$ is a positive definite matrix, while $Q(t)$ and $F$ are positive semidefinite matrices.

For the above system and performance index, the unique optimal controller is given by

$$
u(t)=R(t)^{-1} B(t)^{T}[g(t)-K(t) x(t)]
$$

with $K(t)$ being a $4 \times 4$ positive definite symmetric matrix obtained as the solution of the time-varying Riccati matrix differential equation

$$
\dot{K}(t)=-K(t) A(t)-A(t)^{T} K(t)+K(t) B(t) R^{-1}(t) B(t)^{T} K(t)-C^{T} Q(t) C
$$

with the boundary condition

$$
K(T)=C^{T} F C
$$

The $4 \times 1$ vector $g(t)$ is obtained as the solution of the linear vector differential equation

$$
\dot{g}(t)=-\left[A(t)-B(t) R^{-1}(t) B(t)^{T} K(t)\right]^{T} g(t)-C^{T} Q(t) z(t)
$$


with the boundary condition

$$
g(T)=C^{T} F z(t)
$$

Thus the closed loop system is governed by

$$
\dot{x}(t)=\left[A(t)-B(t) R^{-1}(t) B(t)^{T} K(t)\right] x(t)+B(t) R^{-1}(t) B(t)^{T} g(t)
$$

with given initial condition $x\left(t_{0}\right)$.

We note that Equation (16) is adjoint to Equation (18), i.e. if we use $\Phi\left(t, t_{0}\right)$ to represent the state transition matrix of Equation (18) and $\Psi\left(t, t_{0}\right)$ to represent the state transition matrix of Equation (16), then the relation $\Psi^{\prime}\left(t, t_{0}\right) \Phi\left(t, t_{0}\right)=I$ (where $I$ represents the identity matrix) holds. The optimal control is then given by the expression

$$
u(t)=-R^{-1}(t) B(t)^{T}[K(t) x(t)-g(t)]
$$

For this system, the value of the optimal performance index $J^{*}$ is given by

$$
J^{*}=\frac{1}{2} x(t)^{T} K(t) x(t)-g(t)^{T} x(t)+\phi(t)
$$

where $\phi(t)$ is obtained by a backward integration of the differential equation

$$
\dot{\phi}(t)=-\frac{1}{2}\left[z(t)^{T} Q(t) z(t)-g(t)^{T} B(t) R^{-1} B(t)^{T} g(t)\right]
$$

with boundary condition

$$
\phi(t)=z(T)^{T} K(T) z(T)
$$
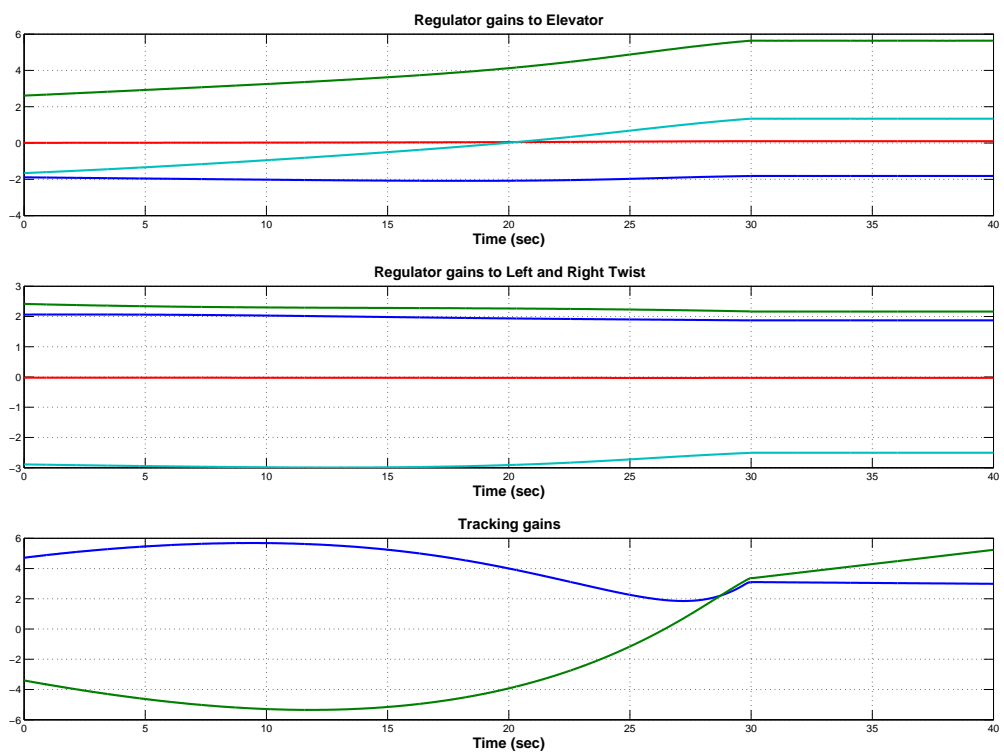

Figure 7. (a)Controller Feedback Gains to Elevator from $V$ (blue), $\alpha$ (green), $q$ (red) and $\theta$ (cyan) (b) Controller Feedback Gains to Twist from $V$ (blue), $\alpha$ (green), $q$ (red) and $\theta$ (cyan) (c) Controller Tracking Inputs to Elevator (blue), Twist (green)

\section{IV.B. Longitudinal Axis Controller}

We first consider the longitudinal axis only; and for this axis, the set of time-varying regulator gains and tracking inputs are demonstrated in Figure 7. The regulator feedback gain matrix is a $2 \times 4$ matrix computed as $R^{-1}(t) B(t)^{T} K(t)$. Figure $7(\mathrm{a})$ represents the regulator feedback gains from the states to the elevator 
V
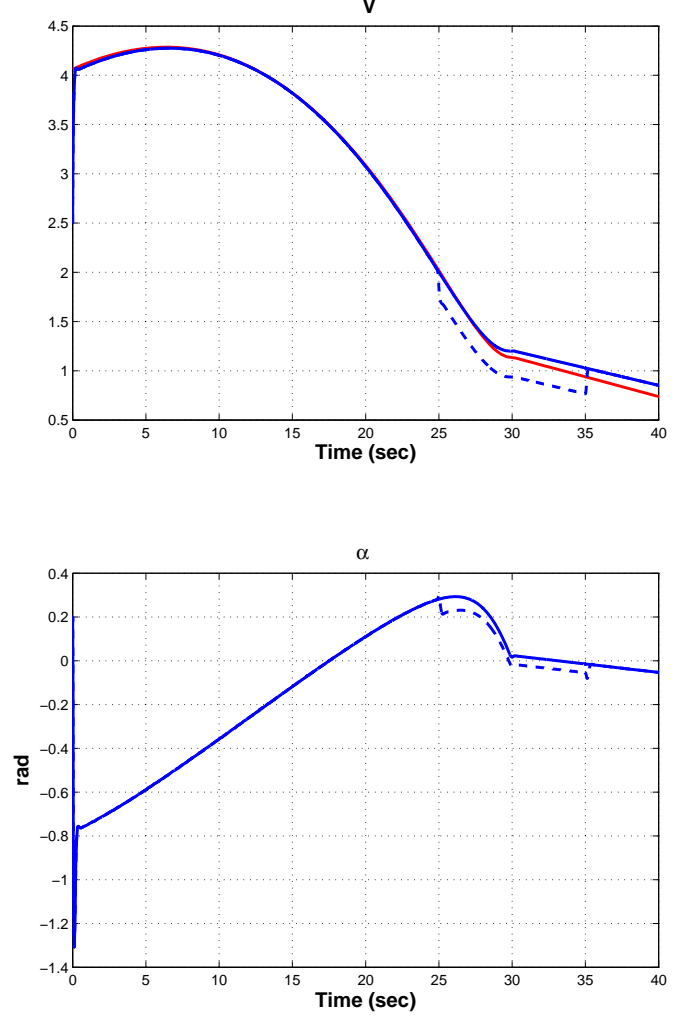
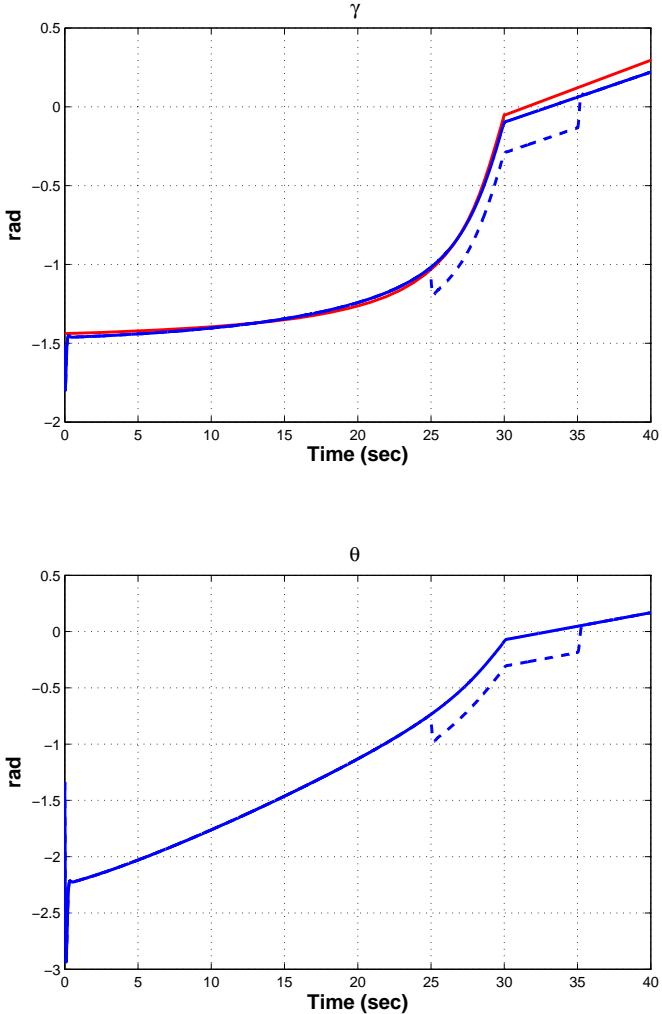

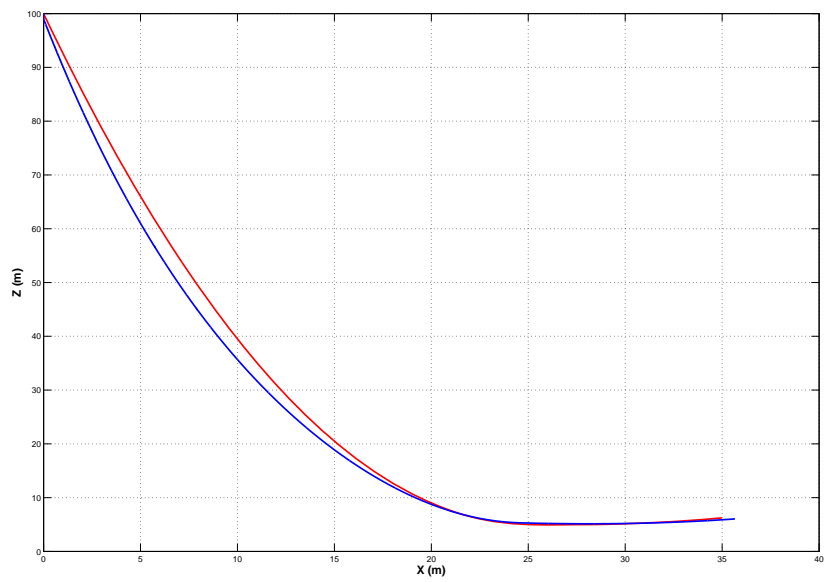

Figure 8. (a-d) Longitudinal axis State responses (e) Perching Trajectory. The reference trajectory is shown in red and the actual trajectory (in the absence of disturbances) is in solid blue. The dashed blue curves indicate the state responses in the presence of disturbance inputs. Note that in (e) the dashed blue is indistinguishable from the solid blue. 
actuator, while Figure 7(b) represents the regulator feedback gains from the states to the left and right wing twist actuators. It is seen that the feedback gain from the velocity state $V$ to the elevator is more or less constant throughout the perching trajectory; while the feedback gains from the states $\alpha$ and $\theta$ vary linearly with time during the dive phase of the trajectory and are subsequently constant during the undershoot and climb phase of the trajectory (which is along expected lines). As far as the feedback gains to the twist actuators are concerned, they are more or less constant with the possible exception of the gain corresponding to the $\theta$ state. All the feedback gains do not change sign during the entire perching trajectory with the exception of the gain from $\theta$ to the elevator. The tracking inputs (which are computed as $R^{-1}(t) B(t)^{T} g(t)$ ) are shown in Figure 7(c) for the elevator and the (left and right) twist actuators respectively. Note that since we are currently considering only the longitudinal axis, therefore the left and right twist actuators have identical values of regulator gains as well as tracking inputs.

Using the gains demonstrated in Figure 7, the closed loop state responses are shown in Figure 8(a-d). Note that these figures show the values of the total state. The reference signals for $V(t)$ and $\gamma(t)$ (as derived from the reference perching trajectory) are shown in red and the actual values of the states are shown in solid blue. Note that (as mentioned in the earlier section), the perching trajectory is actually longitudinally unstable from $t=25 \mathrm{sec}$ onwards, and therefore the controller has performed a reasonable job of both stabilization as well as tracking. To further test the performance of the controller, a persistent disturbance that occurs during the unstable segment of the perching trajectory and for a duration of 10 sec from $t=25$ sec onwards to $t=35 \mathrm{sec}$ is applied. The open-loop system is instantly destabilized, but the closed loop system is able to efficiently reject the effects of the disturbances, as seen in the responses shown with dashed lines. The final values of the flight path angle $\gamma$ and pitch attitude $\theta$ are both positive and the final velocity is less than $1 \mathrm{~m} / \mathrm{sec}$. The controller inputs are demonstrated in Figure 9 both in the absence of disturbances (solid lines) and in the presence of disturbances (dashed lines).
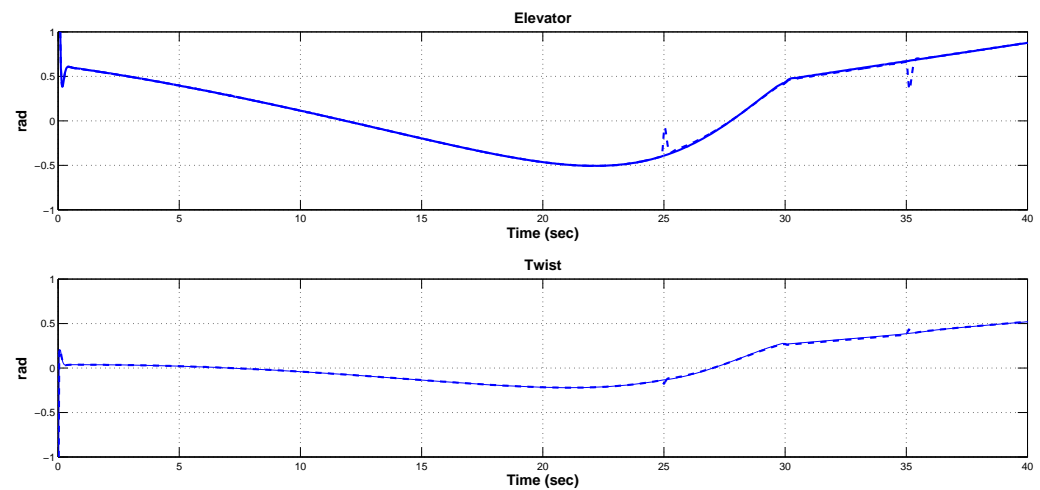

Dihedral

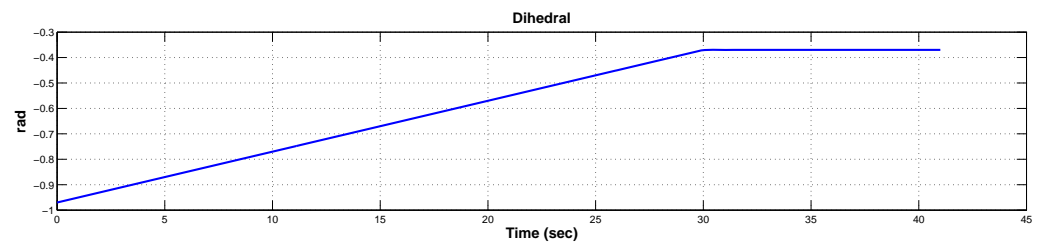

Figure 9. Controller inputs corresponding to (a) Elevator (b) Symmetric Twist (c) Symmetric Dihedral. The solid curves correspond to inputs in the absence of disturbances, while the dashed curves correspond to those in the presence of disturbances.

\section{IV.C. Lateral-Directional Axes Controller}

We next include the effects of lateral-directional dynamics into the aircraft model. Note that (as mentioned earlier) the entire stretch of the perching trajectory is unstable with respect to lateral-directional inputs. Our objective now is to design a lateral controller that will reject any lateral disturbances experienced by the aircraft during the perching trajectory, and then evaluate the resultant deterioration in the actual perching trajectory, when viewed in 3-dimensional space. Note that the outputs of the lateral-directional controller 
are the left and right twist angles. Also, the lateral-directional controller is only of a regulatory nature intended to stabilize the aircraft (as opposed to the longitudinal controller which had both stabilizing as well as tracking functions). The lateral feedback gains are shown in Figure 12. Note that only the gains to the left twist actuator are shown; the gains to the right twist actuator are of the same magnitude of those of the left twist actuator with an opposite sign. The feedback gains are shown in terms of those corresponding to the lateral-directional states $\beta$ (blue), $p$ (green), $r$ (red) and $\phi$ (cyan). It is seen that the gains corresponding to $\beta$ and $\phi$ show the most variation during the perching trajectory. With this controller, the closed loop system is evaluated while giving a persistent disturbance input that affects all the states for a duration of 10 seconds from $t=25 \mathrm{sec}$ to $t=35 \mathrm{sec}$. The open-loop lateral-directional system is instantly destabilized, while the closed loop system is able to utilize asymmetric left and right twist inputs quite effectively to reject the disturbances to the states $\beta, p, r$ and $\phi$, as can be seen from Figure 10(a-d). The actual values of left and right twist input angles generated by the lateral-directional controller are shown in Figure 11(a). The disturbances to these states in turn influence the flight path angle $\gamma$ and $\chi$ (in addition to aircraft heading angle $\psi$ ), as can be seen from the following equations:
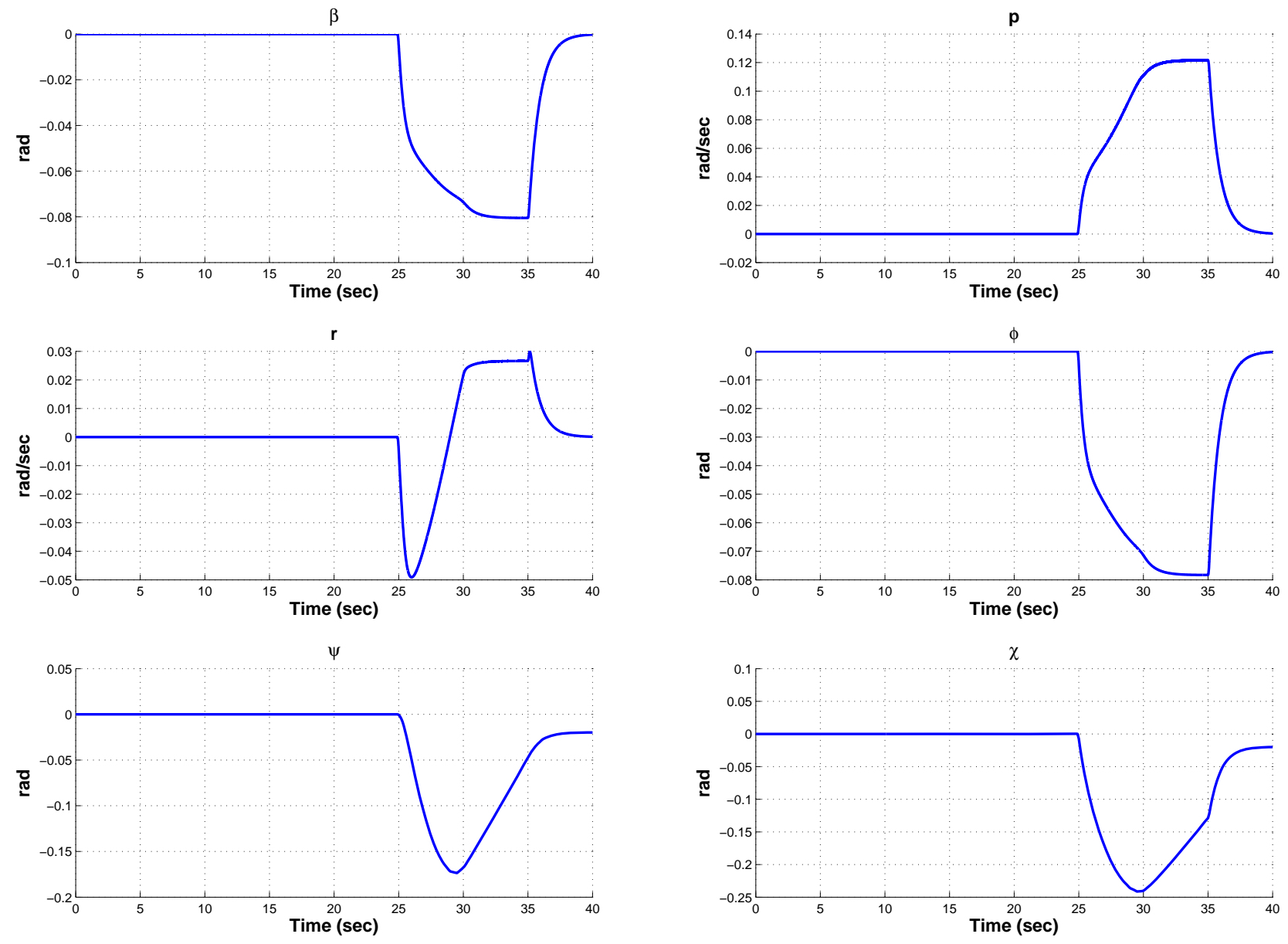

Figure 10. (a-d) Closed loop lateral-directional state responses $\beta, p, r$ and $\phi$ with a disturbance input (e-f) $\psi$ and $\chi$ generated due to lateral disturbances 

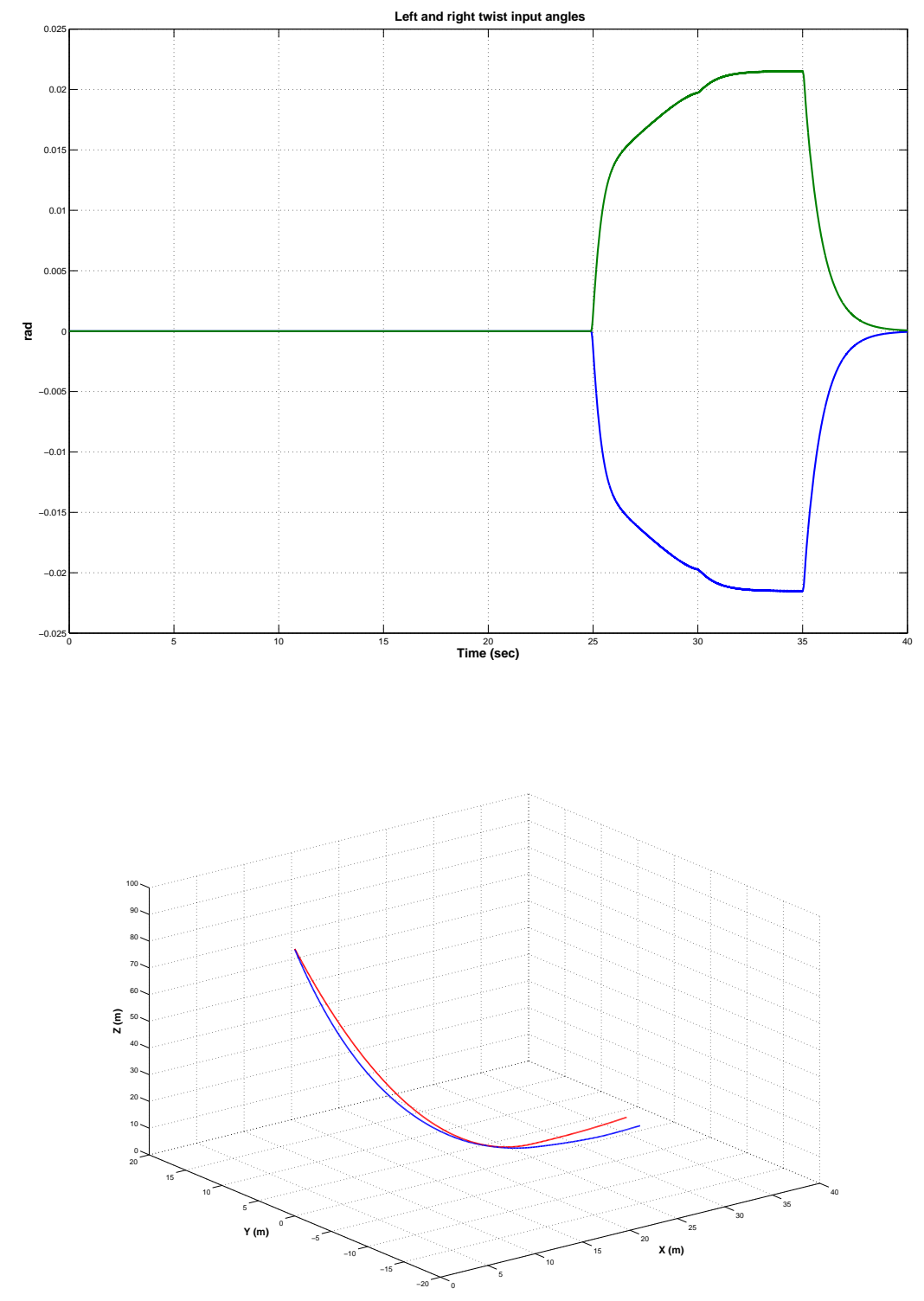

Figure 11. (a) Differential twist inputs generated to reject the lateral disturbance (b) Comparison of reference (red) and true (blue) perching trajectories in three dimensional space 


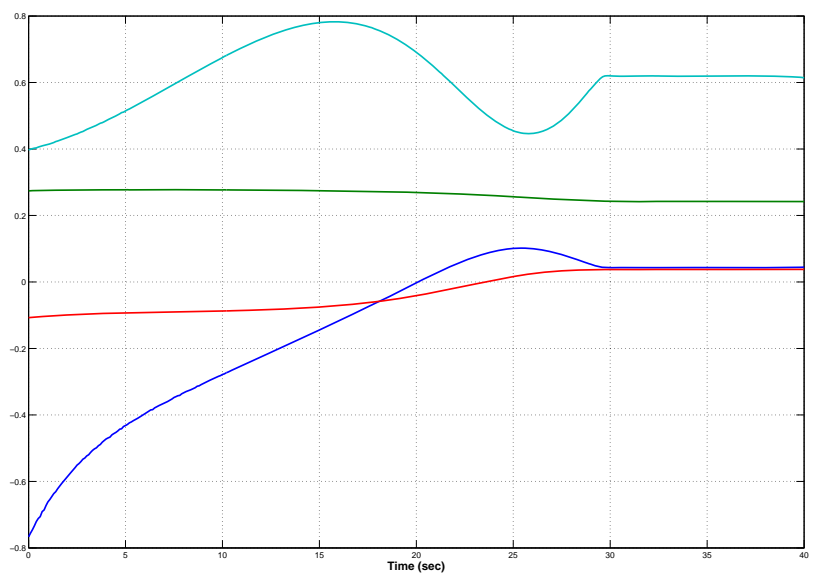

Figure 12. Controller Feedback Gains to Left Twist angle corresponding to $\beta$ (blue), $p$ (green), $r$ (red) and $\phi$ (cyan).

$$
\begin{aligned}
\dot{\psi} & =(q \sin \phi+r \cos \phi) / \cos \theta \\
\sin \gamma & =\cos \alpha \cos \beta \sin \theta-\sin \beta \sin \phi \cos \theta-\sin \alpha \cos \beta \cos \phi \cos \theta \\
\sin \chi \cos \gamma & =\cos \alpha \cos \beta \cos \theta \sin \psi+\sin \beta(\sin \phi \sin \theta \sin \psi+\cos \phi \cos \psi) \\
& +\sin \alpha \cos \beta(\cos \phi \sin \theta \sin \psi-\sin \phi \cos \psi)
\end{aligned}
$$

The closed loop state disturbances of Figures 10(a-d) lead to a neglibibly small change in flight path angle $\gamma$ but do lead to changes of reasonable magnitudes in $\psi$ and $\chi$. These are shown in Figures 10(e-f). From these plots, it is also seen that the steady state values of both $\psi$ and $\chi$ are not exactly zero, but settle at some small non-zero values, which indicate that the final heading of the aircraft at the end of the perching trajectory is slightly displaced from its original intended heading. The ensuing perching trajectory in 3-dimensions (as computed using $\dot{x}(t)=V(t) \cos (\gamma(t)) \cos (\chi(t)), \dot{y}(t)=V(t) \cos (\gamma(t)) \sin (\chi(t)), \dot{z}(t)=V(t) \sin (\gamma(t))$ ) is demonstrated in Figure 11(b), with the reference perching trajectory also shown alongside for purposes of comparison.

\section{Conclusions}

In this paper, the concept of variable wing dihedral and variable wing twist (in conjunction with a conventional horizontal elevator) is effectively used to control an aircraft during a perching trajectory. A specific controller architecture which employs the dihedral (in a symmetric fashion) in the forward path and the elevator and twist in the feedback path, is considered. The wing twist can be used in a symmetric fashion to control the longitudinal response of the aircraft, as well as in an asymmetric fashion to control the lateral-directional response of the aircraft. With this architecture, the aircraft is modeled as a multi-input linear time-varying system. For the specific perching trajectory chosen, it is demonstrated that the open-loop aircraft is longitudinally stable for the initial part of the perching trajectory and unstable for the latter part; and lateral-directionally unstable for the entire perching trajectory. A multi-variable controller with timevarying gains is designed that is able to efficiently stabilize the aircraft as well as reject longitudinal-lateraldirectional wind disturbances, while tracking the reference perching trajectory with fairly high accuracy. Experimental work is currently underway, with the use of a VICON motion capture system. Future work will focus on adapting these controller gains for the nonlinear aircraft model and continuing the experimental validation of the closed loop dynamics of the aircraft. 


\section{Acknowledgements}

This project was supported by the Air Force Office of Scientific Research (AFOSR) under the Young Investigator Award Program (Grant No. FA95500910089) monitored by Dr. W. Larkin. The original problem was posed by Dr. Gregg Abate (AFRL). This paper also benefitted from stimulating discussions with Mr. Johnny Evers (AFRL).

\section{References}

${ }^{1}$ Paranjape, A.A., and Chung, S.-J., "Flight Mechanics of a Tail-less Articulated Wing Aircraft," AIAA Atmospheric Flight Mechanics Conference, Toronto, Canada, August 2010. Invited Paper.

${ }^{2}$ Paranjape, A.A., Chakravarthy, A., Chung, S.-J., and Hilton, H.H., "Performance and Stability of an Agile Tail-less MAV with Flexible Articulated Wings," AIAA Atmospheric Flight Mechanics Conference, Toronto, Canada, August 2010. Invited Paper.

${ }^{3} \mathrm{Ol}$, M., Parker, G., Abate, G., and Evers, J., "Flight Controls and Performance Challenges for MAVs in Complex Environments," AIAA Guidance, Navigation and Control Conference and Exhibit, 2008, AIAA Paper 2008-6508.

${ }^{4}$ Mueller, T.J., "Fixed and Flapping Wing Aerodynamics for Micro Air Vehicle Applications," Progress in Aeronautics and Astronautics, Vol. 195, AIAA, 2001.

${ }^{5}$ Seigler, T.M., Neal, D.A., Bae, J-S., and Inman, D.J., "Modeling and Flight Control of Large-Scale Morphing Aircraft," Journal of Aircraft, Vol. 44, No. 4, July-August 2007, pp. 1077-1087.

${ }^{6}$ Sanders, B., Crowe, R., and Garcia, E., "Defense Advanced Research Projects Agency: Smart Materials and Structures Demonstration Program Overview," Journal of Intelligent Material Systems and Structures, Vol. 15, No. 4, 2004 , pp. $227-233$.

${ }^{7}$ Bowman, J., Sanders, B., and Weisshar, T., "Evaluating the Impact of Morphing Technologies on Aircraft Performance," AIAA Paper 2002-1631, 2002.

${ }^{8}$ Tidwell, Z., Joshi, S., Crossley, W.A., and Ramakrishnan, S., "Comparison of Morphing Wing Strategies Based Upon Aircraft Performance Impacts," AIAA Paper 2004-1722, 2004.

${ }^{9}$ Bye, D.R., and McClure, P.D., "Design of a Morphing Vehicle," AIAA Paper 2007-1728, 2007.

${ }^{10}$ Abdulrahim, M., and Lind, R., "Modeling and Control of Micro Air Vehicles with Biologically-Inspired Morphing," Proceedings of American Control Conference, Minneapolis, Minnesota, June 14-16, 2006, pp. 2718-2723.

${ }^{11}$ Costello, M., and Webb, A., "Wing Articulation of Micro Air Vehicles to Reduce Gust Sensitivity," AIAA Guidance, Navigation and Control Conference and Exhibit, 2008, AIAA Paper 2008-6712.

${ }^{12}$ Wickenheiser, A., and Garcia, E., "Longitudinal Dynamics of a Perching Aircraft," Journal of Aircraft, Vol. 43, No. 5, 2006, pp. 1386-1392.

${ }^{13}$ Wickenheiser, A., and Garcia, E., "Optimization of Perching Maneuvers through Vehicle Morphing," Journal of Guidance, Control and Dynamics, Vol. 31, No. 4, 2008, pp. 815-823.

${ }^{14}$ Reich, G.W., Wojnar, O., and Albertani, R., "Aerodynamic Performance of a Notional Perching MAV Design," 2009, AIAA Paper 2009-63.

${ }^{15}$ Sachs, G., and Holzapfel, F., "Flight Mechanic and Aerodynamic Aspects of Extremely Large Dihedral in Birds," 2007, AIAA Paper 2007-46.

${ }^{16}$ Sachs, G., "What Can Be Learned From Unique Lateral-Directional Dynamics Properties of Birds for Mini-Aircraft," 2007, AIAA Paper 2007-6311.

${ }^{17}$ Sachs, G., "Why Birds and Miniscale Airplanes Need No Vertical Tail," Journal of Aircraft, Vol. 44, No. 4, 2007, pp. 1159-1167.

${ }^{18}$ Dickinson, M.H., Lehmann, F.-O., and Sane, S.P., "Wing Rotation and the Aerodynamic Basis of Insect Flight," Science, Vol. 284, 1999, pp. 1954-1960.

${ }^{19}$ McGahan, J., "Gliding Flight of the Andean Condor in Nature," Journal of Experimental Biology, Vol. 58, No. 1, 1973, pp. 225-237.

${ }^{20}$ Angelo, H.D'.,Linear Time Varying Systems : Analysis and Synthesis, 1970.

${ }^{21}$ Roberts, J.W., Cory, R., and Tedrake, R., "On the Controllability of Fixed-Wing Perching," American Control Conference, St. Louis, MO, June 10-12 2009, pp. 2018-2023.

${ }^{22}$ Desbiens, A.L., and Cutkosky, M.R., "Landing and Perching on Vertical Surfaces with Microspines for Small Unmanned Air Vehicles," Journal of Intelligent Robotic Systems, 57, 2010, pp. 313-327.

${ }^{23}$ Athans, M., and Falb, P., Optimal Control : Theory and Applications, 2007.

${ }^{24}$ Chakravarthy, A., Grant, D.T., and Lind, R., "Time-Varying Dynamics of a Micro Air Vehicle with Variable Sweep Morphing," AIAA Guidance, Navigation and Control Conference, Chicago, August 2009.

${ }^{25}$ Chang, and Chung, S.-J., "Exponential Stability Region Estimates for the State- Dependent Riccati Equation Controllers," Proc. the 48th IEEE Conference on Decision and Control Conference, Shanghai, China, December 2009. 